\title{
Finite Product of Semiring of Sets
}

\author{
Roland Coghetto \\ Rue de la Brasserie 5 \\ 7100 La Louvière, Belgium
}

\begin{abstract}
Summary. We formalize that the image of a semiring of sets [17] by an injective function is a semiring of sets. We offer a non-trivial example of a semiring of sets in a topological space 21. Finally, we show that the finite product of a semiring of sets is also a semiring of sets 21] and that the finite product of a classical semiring of sets [8] is a classical semiring of sets. In this case, we use here the notation from the book of Aliprantis and Border 1 .
\end{abstract}

MSC: 28A05 03E02 03B35

Keywords: set partitions; semiring of sets

MML identifier: SRINGS_4, version: 8.1.04 5.32.1237

The notation and terminology used in this paper have been introduced in the following articles: [9], 2], [3], [4], 22], [7], 15], [23], [10], 11], [6], [12], 20], [26], [27], 19], [14], [16], 25], 18], and [13].

\section{Preliminaries}

From now on $X_{1}, X_{2}, X_{3}, X_{4}$ denote sets.

Now we state the propositions:

(1) (i) $X_{1} \cap X_{4} \backslash\left(X_{2} \cup X_{3}\right)$ misses $X_{1} \backslash\left(\left(X_{2} \cup X_{3}\right) \cup X_{4}\right)$, and

(ii) $X_{1} \cap X_{4} \backslash\left(X_{2} \cup X_{3}\right)$ misses $\left(X_{1} \cap X_{3}\right) \cap X_{4} \backslash X_{2}$, and

(iii) $X_{1} \backslash\left(\left(X_{2} \cup X_{3}\right) \cup X_{4}\right)$ misses $\left(X_{1} \cap X_{3}\right) \cap X_{4} \backslash X_{2}$.

(2) $\left(X_{1} \backslash X_{2}\right) \backslash\left(X_{3} \backslash X_{4}\right)=\left(X_{1} \backslash\left(X_{2} \cup X_{3}\right)\right) \cup\left(X_{1} \cap X_{4} \backslash X_{2}\right)$.

(3) $\left(X_{1} \backslash\left(X_{2} \cup X_{3}\right)\right) \cup\left(X_{1} \cap X_{4} \backslash X_{2}\right)=\left(\left(X_{1} \cap X_{4} \backslash\left(X_{2} \cup X_{3}\right)\right) \cup\left(X_{1} \backslash\left(\left(X_{2} \cup\right.\right.\right.\right.$ $\left.\left.\left.\left.X_{3}\right) \cup X_{4}\right)\right)\right) \cup\left(\left(X_{1} \cap X_{3}\right) \cap X_{4} \backslash X_{2}\right)$. 
(4) $\left(X_{1} \backslash X_{2}\right) \backslash\left(X_{3} \backslash X_{4}\right)=\left(\left(X_{1} \cap X_{4} \backslash\left(X_{2} \cup X_{3}\right)\right) \cup\left(X_{1} \backslash\left(\left(X_{2} \cup X_{3}\right) \cup\right.\right.\right.$ $\left.\left.\left.X_{4}\right)\right)\right) \cup\left(\left(X_{1} \cap X_{3}\right) \cap X_{4} \backslash X_{2}\right)$. The theorem is a consequence of (2) and $(3)$.

(5) $\bigcup\left\{X_{1}, X_{2}, X_{3}\right\}=\left(X_{1} \cup X_{2}\right) \cup X_{3}$.

\section{The Direct Image of a Semiring of Sets by an Injective FUNCTION}

Now we state the proposition:

(6) Let us consider sets $T, S$, a function $f$ from $T$ into $S$, and a family $G$ of subsets of $T$. Then $f^{\circ} G=\left\{f^{\circ} A\right.$, where $A$ is a subset of $\left.T: A \in G\right\}$.

Let $T, S$ be sets, $f$ be a function from $T$ into $S$, and $G$ be a finite family of subsets of $T$. Let us note that $f^{\circ} G$ is finite.

Let $f$ be a function and $A$ be a countable set. Let us note that $f^{\circ} A$ is countable.

The scheme FraenkelCountable deals with a set $\mathcal{A}$ and a set $\mathcal{X}$ and a unary functor $\mathcal{F}$ yielding a set and states that

(Sch. 1) $\{\mathcal{F}(w)$, where $w$ is an element of $\mathcal{A}: w \in \mathcal{X}\}$ is countable

provided

- $\mathcal{X}$ is countable.

Let $T, S$ be sets, $f$ be a function from $T$ into $S$, and $G$ be a countable family of subsets of $T$. Let us note that $f^{\circ} G$ is countable.

Let $X, Y$ be sets, $S$ be a family of subsets of $X$ with the empty element, and $f$ be a function from $X$ into $Y$. One can verify that $f^{\circ} S$ has the empty element.

Now we state the propositions:

(7) Let us consider sets $X, Y$, a function $f$ from $X$ into $Y$, and families $S_{1}, S_{2}$ of subsets of $X$. If $S_{1} \subseteq S_{2}$, then $f^{\circ} S_{1} \subseteq f^{\circ} S_{2}$. The theorem is a consequence of $(6)$.

(8) Let us consider sets $X, Y$, a $\cap$-closed family $S$ of subsets of $X$, and a function $f$ from $X$ into $Y$. Suppose $f$ is one-to-one. Then $f^{\circ} S$ is a $\cap$-closed family of subsets of $Y$.

(9) Let us consider non empty sets $X, Y$, a $\cap_{f p^{-}}$-closed family $S$ of subsets of $X$, and a function $f$ from $X$ into $Y$. Suppose $f$ is one-to-one. Then $f^{\circ} S$ is a $\cap_{f p}$-closed family of subsets of $Y$.

(10) Let us consider non empty sets $X, Y$, a $\backslash \frac{\subset}{f p}$-closed family $S$ of subsets of $X$, and a function $f$ from $X$ into $Y$. Suppose $f$ is one-to-one and $f^{\circ} S$ is not empty. Then $f^{\circ} S$ is a $\backslash \frac{\subset}{f} p$-closed family of subsets of $Y$. 
Proof: Reconsider $f_{1}=f^{\circ} S$ as a family of subsets of $Y$. $f_{1}$ is $\backslash \frac{\subset}{f} p$-closed by [10, (64), (87)], [11, (103)], [26, (123)].

(11) Let us consider non empty sets $X, Y$, a $\backslash_{f}$-closed family $S$ of subsets of $X$, and a function $f$ from $X$ into $Y$. Suppose $f$ is one-to-one. Then $f^{\circ} S$ is

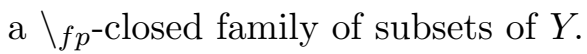

(12) Let us consider non empty sets $X, Y$, a semiring $S$ of sets of $X$, and a function $f$ from $X$ into $Y$. If $f$ is one-to-one, then $f^{\circ} S$ is a semiring of sets of $Y$.

\section{The Set of Set Differences of All Elements of A Semiring of} SETS

Now we state the proposition:

(13) Let us consider a 1-element finite sequence $X$. Suppose $X(1)$ is not empty. Then there exists a function $I$ from $X(1)$ into $\prod X$ such that

(i) $I$ is one-to-one and onto, and

(ii) for every object $x$ such that $x \in X(1)$ holds $I(x)=\langle x\rangle$.

Let $X$ be a set. Observe that $2_{*}^{X}$ is $\cap$-closed and there exists a $\cap$-closed family of subsets of $X$ which has the empty element and there exists a $\cap$-closed family of subsets of $X$ with the empty element which is $\cup$-closed.

Let $X, Y$ be non empty sets. Let us observe that $X \rrbracket Y$ is non empty.

Now we state the proposition:

(14) Let us consider a set $X$, and a family $S$ of subsets of $X$ with the empty element. Then $S \Downarrow S=$ the set of all $A \backslash B$ where $A, B$ are elements of $S$.

Let $X$ be a set and $S$ be a family of subsets of $X$ with the empty element. The functor semidiff $S$ yielding a family of subsets of $X$ is defined by the term (Def. 1) $S \Downarrow S$.

Now we state the proposition:

(15) Let us consider a set $X$, a family $S$ of subsets of $X$ with the empty element, and an object $x$. Suppose $x \in \operatorname{semidiff} S$. Then there exist elements $A, B$ of $S$ such that $x=A \backslash B$. The theorem is a consequence of (14).

Let $X$ be a set and $S$ be a family of subsets of $X$ with the empty element. Observe that semidiff $S$ has the empty element.

Let $S$ be a $\cap$-closed, $\cup$-closed family of subsets of $X$ with the empty element. Note that semidiff $S$ is $\cap$-closed and $\backslash f p^{-c l o s e d}$.

Now we state the proposition:

(16) Let us consider a set $X$, and a $\cap$-closed, $\cup$-closed family $S$ of subsets of $X$ with the empty element. Then semidiff $S$ is a semiring of sets of $X$. 
4. The Collection of All Locally Closed Sets $L C(X, \tau)$ of A Topological Space $(X, \tau)$

Let $T$ be a non empty topological space. The functor $\mathrm{LC}(T)$ yielding a family of subsets of $\Omega_{T}$ is defined by the term

(Def. 2) $\{A \cap B$, where $A, B$ are subsets of $T: A$ is open and $B$ is closed $\}$.

Let us note that $\mathrm{LC}(T)$ is $\cap$-closed and $\backslash{ }_{f p}$-closed and has the empty element.

(17) Let us consider a non empty topological space $T$. Then $\operatorname{LC}(T)$ is a semiring of sets of $\Omega_{T}$.

\section{The Finite Product of Semirings of Sets}

Let $n$ be a natural number. Note that there exists an $n$-element finite sequence which is non-empty.

Let $n$ be a non zero natural number and $X$ be a non-empty, $n$-element finite sequence.

A semiring family of $X$ is an $n$-element finite sequence and is defined by

(Def. 3) for every natural number $i$ such that $i \in \operatorname{Seg} n$ holds $i t(i)$ is a semiring of sets of $X(i)$.

In the sequel $n$ denotes a non zero natural number and $X$ denotes a nonempty, $n$-element finite sequence. Now we state the propositions:

(18) Let us consider a semiring family $S$ of $X$. Then $\operatorname{dom} S=\operatorname{dom} X$.

(19) Let us consider a semiring family $S$ of $X$, and a natural number $i$. If $i \in \operatorname{Seg} n$, then $\bigcup(S(i)) \subseteq X(i)$.

(20) Let us consider a function $f$, and an $n$-element finite sequence $X$. If $f \in \prod X$, then $f$ is an $n$-element finite sequence.

Let $n$ be a non zero natural number and $X$ be an $n$-element finite sequence. The functor SemiringProduct $X$ yielding a set is defined by

(Def. 4) for every object $f, f \in i t$ iff there exists a function $g$ such that $f=\prod g$ and $g \in \prod X$.

Now we state the propositions:

(21) Let us consider an $n$-element finite sequence $X$. Then SemiringProduct $X \subseteq 2^{(\cup \cup X)^{\operatorname{dom} X}}$.

(22) Let us consider a semiring family $S$ of $X$. Then SemiringProduct $S$ is a family of subsets of $\prod X$.

Proof: Reconsider $S_{1}=$ SemiringProduct $S$ as a subset of $2^{(\bigcup \bigcup S)^{\text {dom } S}}$. $S_{1} \subseteq 2 \prod^{X}$ by [3, (9)], (18), [7, (89)], (19). 
(23) Let us consider a non-empty, 1-element finite sequence $X$. Then $\prod X=$ the set of all $\langle x\rangle$ where $x$ is an element of $X(1)$. The theorem is a consequence of (13).

One can check that $\Pi\langle\emptyset\rangle$ is empty. Now we state the propositions:

(24) Let us consider a non empty set $x$. Then $\prod\langle x\rangle=$ the set of all $\langle y\rangle$ where $y$ is an element of $x$. The theorem is a consequence of (23).

(25) Let us consider a non-empty, 1-element finite sequence $X$, and a semiring family $S$ of $X$. Then SemiringProduct $S=$ the set of all $\Pi\langle s\rangle$ where $s$ is an element of $S(1)$. Proof: $S$ is non-empty by (18), [7, (3)]. $\prod S=$ the set of all $\langle s\rangle$ where $s$ is an element of $S(1)$.

Let us consider sets $x, y$. Now we state the propositions:

(26) $\prod\langle x\rangle \cap \prod\langle y\rangle=\prod\langle x \cap y\rangle$. The theorem is a consequence of (24).

(27) $\Pi\langle x\rangle \backslash \Pi\langle y\rangle=\prod\langle x \backslash y\rangle$. The theorem is a consequence of (24).

Let us consider a non-empty, 1-element finite sequence $X$ and a semiring family $S$ of $X$. Now we state the propositions:

(28) the set of all $\prod\langle s\rangle$ where $s$ is an element of $S(1)$ is a semiring of sets of the set of all $\langle x\rangle$ where $x$ is an element of $X(1)$. The theorem is a consequence of (24), (26), and (27).

(29) SemiringProduct $S$ is a semiring of sets of $\prod X$. The theorem is a consequence of (23), (25), and (28).

(30) Let us consider sets $X_{1}, X_{2}$, a semiring $S_{1}$ of sets of $X_{1}$, and a semiring $S_{2}$ of sets of $X_{2}$. Then the set of all $s_{1} \times s_{2}$ where $s_{1}$ is an element of $S_{1}$, $s_{2}$ is an element of $S_{2}$ is a semiring of sets of $X_{1} \times X_{2}$.

(31) Let us consider a non-empty, $n$-element finite sequence $X_{3}$, a non-empty, 1-element finite sequence $X_{1}$, a semiring family $S_{3}$ of $X_{3}$, and a semiring family $S_{1}$ of $X_{1}$. Suppose SemiringProduct $S_{3}$ is a semiring of sets of $\prod X_{3}$ and SemiringProduct $S_{1}$ is a semiring of sets of $\prod X_{1}$. Let us consider a family $S_{4}$ of subsets of $\prod X_{3} \times \prod X_{1}$. Suppose $S_{4}=$ the set of all $s_{1} \times$ $s_{2}$ where $s_{1}$ is an element of SemiringProduct $S_{3}, s_{2}$ is an element of SemiringProduct $S_{1}$. Then there exists a function $I$ from $\prod X_{3} \times \prod X_{1}$ into $\prod\left(X_{3} \frown X_{1}\right)$ such that

(i) $I$ is one-to-one and onto, and

(ii) for every finite sequences $x, y$ such that $x \in \prod X_{3}$ and $y \in \prod X_{1}$ holds $I(x, y)=x^{\frown} y$, and

(iii) $I^{\circ} S_{4}=\operatorname{SemiringProduct}\left(S_{3} \frown S_{1}\right)$.

Proof: $\bigcup\left(S_{1}(1)\right) \subseteq X_{1}(1)$. Consider $I$ being a function from $\prod X_{3} \times \prod X_{1}$ into $\prod\left(X_{3} \frown X_{1}\right)$ such that $I$ is one-to-one and $I$ is onto and for every finite 
sequences $x, y$ such that $x \in \prod X_{3}$ and $y \in \prod X_{1}$ holds $I(x, y)=x^{\frown} y$. $I^{\circ} S_{4}=\operatorname{Semiring} \operatorname{Product}\left(S_{3} \frown S_{1}\right)$ by (25), (20), [7, (89)], [24, (153)].

(32) Let us consider a non-empty, $n$-element finite sequence $X_{3}$, a non-empty, 1-element finite sequence $X_{1}$, a semiring family $S_{3}$ of $X_{3}$, and a semiring family $S_{1}$ of $X_{1}$. Suppose SemiringProduct $S_{3}$ is a semiring of sets of $\prod X_{3}$ and SemiringProduct $S_{1}$ is a semiring of sets of $\prod X_{1}$. Then SemiringProduct $\left.\left(S_{3}\right\urcorner S_{1}\right)$ is a semiring of sets of $\prod\left(X_{3} \frown X_{1}\right)$. The theorem is a consequence of (30), (31), (9), and (10).

(33) Let us consider a semiring family $S$ of $X$. Then SemiringProduct $S$ is a semiring of sets of $\prod X$. Proof: Define $\mathcal{P}$ [non zero natural number] $\equiv$ for every non-empty, $\$_{1}$-element finite sequence $X$ for every semiring family $S$ of $X$, SemiringProduct $S$ is a semiring of sets of $\prod X$. $\mathcal{P}[1]$. For every non zero natural number $n, \mathcal{P}[n]$ from [5, Sch. 10].

Let $n$ be a non zero natural number, $X$ be a non-empty, $n$-element finite sequence, and $S$ be a semiring family of $X$. We say that $S$ is $\cap$-closed yielding if and only if

(Def. 5) for every natural number $i$ such that $i \in \operatorname{Seg} n$ holds $S(i)$ is $\cap$-closed.

Note that there exists a semiring family of $X$ which is $\cap$-closed yielding.

\section{The Finite Product of Classical Semirings of Sets}

Let $X$ be a set. Note that there exists a semiring of sets of $X$ which is $\cap$-closed.

Let us consider a non-empty, 1-element finite sequence $X$ and a $\cap$-closed yielding semiring family $S$ of $X$. Now we state the propositions:

(34) the set of all $\prod\langle s\rangle$ where $s$ is an element of $S(1)$ is a $\cap$-closed semiring of sets of the set of all $\langle x\rangle$ where $x$ is an element of $X(1)$. The theorem is a consequence of (26) and (28).

(35) SemiringProduct $S$ is a $\cap$-closed semiring of sets of $\prod X$. The theorem is a consequence of (23), (25), and (34).

Now we state the propositions:

(36) Let us consider sets $X_{1}, X_{2}$, a $\cap$-closed semiring $S_{1}$ of sets of $X_{1}$, and a $\cap$-closed semiring $S_{2}$ of sets of $X_{2}$. Then the set of all $s_{1} \times s_{2}$ where $s_{1}$ is an element of $S_{1}, s_{2}$ is an element of $S_{2}$ is a $\cap$-closed semiring of sets of $X_{1} \times X_{2}$.

(37) Let us consider a non-empty, $n$-element finite sequence $X_{3}$, a non-empty, 1-element finite sequence $X_{1}$, a $\cap$-closed yielding semiring family $S_{3}$ of $X_{3}$, and a $\cap$-closed yielding semiring family $S_{1}$ of $X_{1}$. Suppose SemiringProduct 
$S_{3}$ is a $\cap$-closed semiring of sets of $\prod X_{3}$ and SemiringProduct $S_{1}$ is a $\cap$-closed semiring of sets of $\prod X_{1}$. Then $\operatorname{SemiringProduct}\left(S_{3} \frown S_{1}\right)$ is a $\cap$-closed semiring of sets of $\prod\left(X_{3} \frown X_{1}\right)$. The theorem is a consequence of $(30),(31),(36),(8)$, and (10).

Let us consider $n$ and $X$. Let $S$ be a $\cap$-closed yielding semiring family of $X$. One can check that SemiringProduct $S$ is $\cap$-closed.

(38) Let us consider a $\cap$-closed yielding semiring family $S$ of $X$. Then SemiringProduct $S$ is a $\cap$-closed semiring of sets of $\prod X$.

\section{Measurable Rectangle}

Let $n$ be a non zero natural number and $X$ be a non-empty, $n$-element finite sequence.

A classical semiring family of $X$ is an $n$-element finite sequence and is defined by

(Def. 6) for every natural number $i$ such that $i \in \operatorname{Seg} n$ holds $i t(i)$ is a semi-diffclosed, $\cap$-closed family of subsets of $X(i)$ with the empty element.

Let $X$ be an $n$-element finite sequence. We introduce MeasurableRectangle $X$ as a synonym of SemiringProduct $X$. Now we state the propositions:

(39) Every classical semiring family of $X$ is a $\cap$-closed yielding semiring family of $X$.

(40) Let us consider a classical semiring family $S$ of $X$.

Then MeasurableRectangle $S$ is a semi-diff-closed, $\cap$-closed family of subsets of $\prod X$ with the empty element. The theorem is a consequence of (39) and (33).

ACKnowledgement: I would like to thank Artur Korniłowicz for suggestions.

\section{REFERENCES}

[1] Charalambos D. Aliprantis and Kim C. Border. Infinite dimensional analysis. SpringerVerlag, Berlin, Heidelberg, 2006.

[2] Grzegorz Bancerek. Cardinal numbers Formalized Mathematics, 1(2):377-382, 1990.

[3] Grzegorz Bancerek. König's theorem. Formalized Mathematics, 1(3):589-593, 1990.

[4] Grzegorz Bancerek. Tarski's classes and ranks. Formalized Mathematics, 1(3):563-567, 1990.

[5] Grzegorz Bancerek. The fundamental properties of natural numbers Formalized Mathematics, 1(1):41-46, 1990.

[6] Grzegorz Bancerek. The ordinal numbers Formalized Mathematics, 1(1):91-96, 1990.

[7] Grzegorz Bancerek and Krzysztof Hryniewiecki. Segments of natural numbers and finite sequences. Formalized Mathematics, 1(1):107-114, 1990. 
[8] Vladimir Igorevich Bogachev and Maria Aparecida Soares Ruas. Measure theory, volume 1. Springer, 2007.

[9] Czesław Byliński. Binary operations. Formalized Mathematics, 1(1):175-180, 1990.

[10] Czesław Byliński. Functions and their basic properties Formalized Mathematics, 1(1): 55-65, 1990.

[11] Czesław Byliński. Functions from a set to a set Formalized Mathematics, 1(1):153-164, 1990.

[12] Czesław Byliński. Partial functions. Formalized Mathematics, 1(2):357-367, 1990.

[13] Czesław Byliński. Some basic properties of sets Formalized Mathematics, 1(1):47-53, 1990.

[14] Roland Coghetto. Semiring of sets. Formalized Mathematics, 22(1):79-84, 2014. doi:10.2478/forma-2014-0008.

[15] Agata Darmochwał. Finite sets. Formalized Mathematics, 1(1):165-167, 1990.

[16] Noboru Endou, Kazuhisa Nakasho, and Yasunari Shidama. $\sigma$-ring and $\sigma$-algebra of sets. Formalized Mathematics, 23(1):51-57, 2015. doi 10.2478/forma-2015-0004.

[17] D.F. Goguadze. About the notion of semiring of sets. Mathematical Notes, 74:346-351, 2003. ISSN 0001-4346. doi 10.1023/A:1026102701631.

[18] Zbigniew Karno. On discrete and almost discrete topological spaces Formalized Mathematics, 3(2):305-310, 1992.

[19] Beata Padlewska. Families of sets Formalized Mathematics, 1(1):147-152, 1990.

[20] Beata Padlewska and Agata Darmochwał. Topological spaces and continuous functions Formalized Mathematics, 1(1):223-230, 1990.

[21] Jean Schmets. Théorie de la mesure Notes de cours, Université de Liège, 146 pages, 2004.

[22] Andrzej Trybulec. Enumerated sets. Formalized Mathematics, 1(1):25-34, 1990.

[23] Andrzej Trybulec and Agata Darmochwał. Boolean domains. Formalized Mathematics, 1 (1):187-190, 1990.

[24] Wojciech A. Trybulec. Non-contiguous substrings and one-to-one finite sequences Formalized Mathematics, 1(3):569-573, 1990.

[25] Zinaida Trybulec. Properties of subsets Formalized Mathematics, 1(1):67-71, 1990.

[26] Edmund Woronowicz. Relations and their basic properties. Formalized Mathematics, 1 (1):73-83, 1990.

[27] Edmund Woronowicz. Relations defined on sets. Formalized Mathematics, 1(1):181-186, 1990. 